\title{
Partial sequence comparison of the rpoB, sod $A$, groEL and gyrB genes within the genus Streptococcus
}

Correspondence

Véronique Roux

vroux91@hotmail.com
Olga O. Glazunova, Didier Raoult and Véronique Roux

Laboratoire de Bactériologie - Virologie, Hôpital de la Timone, CNRS UMR 6236, IFR48, 264 rue Saint-Pierre, 13385 Marseille, Cedex 05, France

\begin{abstract}
Phylogenetic analysis and species identification of members of the genus Streptococcus were carried out using partial sequence comparison of the 16S rRNA gene (1468-1478 bp), rpoB, encoding the $\beta$ subunit of RNA polymerase (659-680 bp), sod $A$, encoding the manganesedependent superoxide dismutase (435-462 bp), groEL, encoding the $60 \mathrm{kDa}$ heat-shock protein (757 bp), and gyrB, encoding the B subunit of DNA gyrase (458-461 bp). For the first time, most species within the genus Streptococcus were represented in the study (65 strains, representing 58 species and nine subspecies). Phylogenies inferred from $r p o B$, sodA, gyrB and groEL sequence comparisons were more discriminative than those inferred from 16S rRNA gene sequence comparison, and showed common clusters. The minimal interspecies divergence was $0.3,2.7,0,2.5$ and $3.4 \%$ for the $16 \mathrm{~S}$ rRNA gene, $r p o B$, sod $A$, gyrB and groEL, respectively. In general, groEL partial gene sequence comparison represented the best tool for identifying species and subspecies and for phylogenetic analysis.
\end{abstract}

The streptococci are non-motile, Gram-positive cocci that are a part of the family Streptococcaceae. Currently, 66 species and 12 subspecies are recognized as members of the genus Streptococcus (http://www.bacterio.cict.fr/s/streptococcus.html). Until recently, streptococci were identified based only on the haemolytic reaction, group carbohydrate antigens and phenotypic tests (Facklam, 2002). Phylogenetic analysis and identification of representatives of the genus Streptococcus has been based on 16S rRNA gene sequence comparisons (Bentley et al., 1991), but the lack of variability of the $16 \mathrm{~S}$ rRNA gene sequence did not allow identification of closely related species or subspecies. Other genes have subsequently been used (Garnier et al., 1997; Kawamura et al., 1999, 2005; Igarashi et al., 2001; Teng et al., 2002; Tapp et al., 2003; Picard et al., 2004; Hung et al., 2005).

In order to discriminate among species, a gene must be present in a single copy, widely distributed among bacterial genomes and sufficiently variable to differentiate species of the considered genus (Zeigler, 2003). We chose to complete the work initiated by Poyart et al. (1998), Drancourt et al. (2004) and Hoshino et al. (2005), and determined 16S

The GenBank/EMBL/DDBJ accession numbers for the sequences determined in this study are detailed in Supplementary Table S1.

Details of primers, strains and sequence accession numbers, neighbourjoining trees derived from $16 \mathrm{~S}$ rRNA gene, $\operatorname{sod} A, r p o B$ and gyrB sequences and a representation of the distribution of pairwise similarity for each gene are available as supplementary material with the online version of this paper.
rRNA gene sequences for streptococcal type strains that were not represented in GenBank and sequenced gyrB and groEL gene fragments. During the course our study, Itoh et al. (2006) published gyrB gene sequences for 37 species. In our study, most recognized species of the genus Streptococcus were included, for the first time. We compared the sequence data for each gene for phylogenetic analysis and to evaluate the discriminatory power of the individual genes in identification of streptococci.

We studied 65 streptococcal strains (representing 58 species and nine subspecies; see Supplementary Table S1, available in IJSEM Online), mostly obtained from the Pasteur Institute Collection (CIP, Paris, France). Bacterial DNA was extracted using the MagNA Pure LC DNA isolation kit III (Roche) with the MagNA Pure LC instrument as described by the manufacturer. PCR amplification of the $16 \mathrm{~S}$ rRNA gene was performed using the universal primer pair fD1 and rp2 (Weisburg et al., 1991). The primer pair $d 1$ and $d 2$ (Poyart et al., 1998) and primers StreptoF and StreptoR (Drancourt et al., 2004) were respectively used for PCR amplification of $\operatorname{sod} A$ and $r p o B$, as described previously. The locations of the amplification primers for groEL and $g y r B$ gene fragments (streptogroELd/streptogroELr and streptogyrBd/ streptogyrBr) were chosen in conserved regions. Primers are described in Supplementary Table S2. At the outset of this study, we defined these conserved regions by aligning all the available genome sequences for streptococci [Streptococcus pneumoniae strains R6 (GenBank accession 
no. NC_003098) and TIGR4 (NC_003028), Streptococcus agalactiae strains NEM316 (NC_004368) and 2603V/R (NC_004116), Streptococcus pyogenes strains M1 GAS (NC_002737) and MGAS315 (NC_004070), Streptococcus mutans UA159 (NC_004350) and Streptococcus thermophilus strains LMG 18311 (NC_006448) and CNRZ1066 (NC_006449)] and other phylogenetically related bacteria [Lactococcus lactis subsp. lactis II1403 (NC_002662), Listeria innocua Clip11262 (NC_003212) and Listeria monocytogenes EGD-e (NC_003210)]. For groEL, the sequences determined by Teng et al. (2002) and Hung et al. (2005) were also included in the alignment. The annealing temperature for the PCR was $52{ }^{\circ} \mathrm{C}$. No product was obtained for gyrB with the chosen primer pair for the type strain of Streptococcus suis; another pair of primers was designed in a conserved downstream region (GyrBsuisd and GyrBsuisr), and a PCR product was obtained using StreptogyrBd and GyrBsuisr. Other primers were designed for sequencing reactions. PCR products were purified using MultiScreen PCR plates (Millipore) and sequenced using a DNA sequencing kit (BigDye Terminator v. 1.1 cycle sequencing kit; PE Biosystems) according to the manufacturer's instructions. Sequence products were purified and electrophoresis was performed with an Applied Biosystems 3100 Genetic Analyzer. The sequences were submitted to GenBank, and accession numbers are indicated in Supplementary Table S1. Gene sequences were aligned using the multi-sequence alignment program CLUSTAL_X 1.8 (Thompson et al., 1997). Phylogenetic relationships were determined by using MEGA version 4 (Tamura et al., 2007). Distance matrices were determined following the assumptions described by Kimura (1980) and were used to elaborate dendrograms using the neighbour-joining method (Saitou \& Nei, 1987). The maximum-parsimony algorithm (Farris, 1970) and the maximum-likelihood method using the program DNAML in the PHYLIP software package (Felsenstein, 1989) were also used for phylogenetic analysis. Bootstrap values were obtained for a consensus tree based on 100 randomly generated trees.

The genes studied represented different COG functional categories: replication, recombination and repair genes (gyrB; COG0187), transcription genes (rpoB, COG0085), post-translational modification, protein turnover and chaperone genes (groEL; COG0459) and inorganic ion transport and metabolism genes (sodA; COG0605). Moreover, three of the four chosen genetic targets have been considered as part of the 'minimal gene-set' (i.e. genes necessary to maintain a living cell; Gil et al., 2004). These are distributed in different categories: DNA metabolism $(g y r B)$, RNA metabolism $(r p o B)$ and protein processing, folding and secretion ( $g r o E L)$.

Phylogenies were inferred from the five analysed genes and from the $16 \mathrm{~S}$ rRNA gene. Identical topologies were obtained for each gene using the neighbour-joining, maximum-parsimony and maximum-likelihood methods; however, the highest bootstrap values were obtained with the neighbour-joining method, and it is these values that are referred to in the discussion. We considered only clusters supported by bootstrap values greater than $85 \%$.

A PCR fragment of 1468-1478 bp was amplified from the $16 \mathrm{~S}$ rRNA gene. In the 'mitis' group, five species grouped together with a bootstrap value of $91 \%$ (S. pneumoniae, S. pseudopneumoniae, S. mitis, S. oralis and S. infantis) (Supplementary Fig. S1). Streptococcus oligofermentans and $S$. sinensis clustered together with a significant bootstrap value of $99 \%$. Seven other clusters were noted: (i) the 'salivarius' group (Streptococcus thermophilus, S. vestibularis and S. salivarius); (ii) representatives of the 'equinus' group (Streptococcus gallolyticus subsp. gallolyticus, S. gallolyticus subsp. pasteurianus, S. gallolyticus subsp. macedonicus, S. infantarius subsp. infantarius, S. infantarius subsp. coli and S. equinus); (iii) Streptococcus downei and S. sobrinus; (iv) S. pyogenes and S. canis; (v) Streptococcus intermedius and S. constellatus; (vi) Streptococcus parauberis and S. iniae; and (vii) the 'hyovaginalis' group (Streptococcus hyovaginalis, S. pluranimalium and $S$. thoraltensis). All the other species clustered with non-significant bootstrap values at the nodes.

A fragment of $435 \mathrm{bp}$ was obtained for all the streptococci except Streptococcus massiliensis (462 bp) for sodA. The PCR fragment represented $69.5 \%$ of the sodA gene sequence. All the representatives of the 'mitis' group clustered with a bootstrap value of $97 \%$ (Supplementary Fig. S2a). Three subclusters were found: the first included Streptococcus anginosus, S. intermedius and S. constellatus (bootstrap value $99 \%$ ), the second included Streptococcus sanguinis and S. gordonii (bootstrap value $87 \%$ ) and the third included S. infantis, S. australis, S. peroris, S. oralis, S. pneumoniae, S. pseudopneumoniae, S. mitis and S. oligofermentans (bootstrap value $99 \%$ ). Six other clusters were noted: (i) the 'salivarius' group; (ii) the 'equinus' group; (iii) the 'hyovaginalis' group; (iv) the 'criceti' group (Streptococcus criceti, S. downei and S. sobrinus); (v) the 'pyogenes' group (S. canis, S. pyogenes, S. dysgalactiae subsp. dysgalactiae and $S$. dysgalactiae subsp. equisimilis); and (vi) Streptococcus devriesei and S. ratti. All the other species clustered with non-significant bootstrap values at the nodes.

A fragment of $680 \mathrm{bp}$ was amplified and sequenced for $r p o B$ for all species except Streptococcus marimammalium (659 bp). The PCR fragment represented $18.2 \%$ of the fulllength $r p o B$ gene sequence. In the 'mitis' group, seven species were grouped together with a significant bootstrap value of $100 \%$ (S. infantis, S. peroris, S. oralis, S. oligofermentans, S. mitis, S. pneumoniae and S. pseudopneumoniae) (Supplementary Fig. S2b). Streptococcus constellatus, $S$. anginosus and $S$. intermedius formed one monophyletic cluster and S. sinensis, S. sanguinis and $S$. gordonii formed another, with respective bootstrap values of 100 and $98 \%$. S. australis and S. parasanguinis clustered together with a bootstrap value of $100 \%$. Five other 
clusters were noted: (i) the 'salivarius' group; (ii) the 'equinus' group; (iii) the 'hyovaginalis' group; (iv) the 'criceti' group; and (v) the 'pyogenes' group. Three pairs of species grouped together with significant bootstrap values: (i) Streptococcus ovis and S. minor; (ii) S. devriesei and S. ratti; and (iii) S. suis and S. gallinaceus. All the other species clustered with non-significant bootstrap values at the nodes.

Primers used to amplify gyrB generated a $458 \mathrm{bp}$ fragment (representing $23.5 \%$ of the whole gene sequence) for all species except Streptococcus ferus (461 bp). The representatives of the 'mitis' group were separated into several clusters (Supplementary Fig. S2c). The first included $S$. constellatus, S. intermedius and S. anginosus (bootstrap value $100 \%$ ), the second included S. massiliensis, S. sinensis, S. sanguinis and $S$. cristatus (bootstrap value $90 \%$ ) and the third included S. infantis, S. peroris, S. oralis, $S$. oligofermentans, $S$. mitis, $S$. pneumoniae and $S$. pseudopneumoniae (bootstrap value $100 \%$ ). Another cluster including S. gallinaceus, S. ovis, S. minor, S. acidominimus and S. suis (bootstrap value $42 \%$ ) split the 'mitis' group streptococci. Using the maximum-parsimony method, S. australis clustered with the 'minor' subcluster, but, using the neighbour-joining and maximum-likelihood methods, S. australis clustered with the 'sanguinis' subcluster. Five other clusters were noted: (i) the 'salivarius' group; (ii) the 'hyovaginalis' group; (iii) the 'criceti' group; (iv) the 'pyogenes' group and Streptococcus castoreus; and (v) S. devriesei, S. ratti and S. mutans. For the representatives of the 'equinus' group, two subgroups were noted: the first included the subspecies of S. gallolyticus and the second included $S$. equinus and the subspecies of $S$. infantarius. Streptococcus alactolyticus was closely related to the latter species, but the bootstrap value for the node was not significant $(70 \%)$. All the other species clustered with non-significant bootstrap values at the nodes.

Previously, Itoh et al. (2006) performed a phylogenetic analysis of 37 species of the genus Streptococcus based on sequence comparison of a $900 \mathrm{bp}$ gyrB gene fragment. Many clusters were the same in our study: the 'salivarius' group, the 'criceti' group, S. mutans and S. ratti, the 'pyogenes' group and Streptococcus phocae, and S. hyovaginalis and S. thoraltensis. S. equinus and S. gallolyticus clustered together. All the species of the 'mitis' group clustered together with an organization similar to that found in our study, but more species were included in our study.

In all studied strains, a $757 \mathrm{bp}$ fragment was amplified for groEL, representing $46.6 \%$ of the full-length sequence. All the representatives of the 'mitis' group clustered with a bootstrap value of $86 \%$ (Fig. 1). S. acidominimus and $S$. suis were also included in this cluster. Two subclusters were observed: the first included S. constellatus, S. anginosus, $S$. intermedius, S. massiliensis, S. sanguinis, S. gordonii, S. cristatus and S. sinensis (bootstrap value $89 \%$ ) and the second included S. australis, S. parasanguinis, S. peroris, $S$. infantis, S. oralis, S. oligofermentans, S. mitis, S. pneumoniae and S. pseudopneumoniae (bootstrap value $94 \%$ ). Six other clusters were noted: (i) the 'salivarius' group; (ii) the 'equinus' group; (iii) the 'hyovaginalis' group; (iv) the 'criceti' group; (v) the 'pyogenes' group and S. castoreus; and (vi) S. devriesei, S. ratti, S. mutans and S. macacae. All the other species clustered with non-significant bootstrap values at the nodes.

A phylogenetic analysis based on groEL gene sequence comparison was performed by Teng et al. (2002) for viridans group streptococci ( $S$. constellatus, S. anginosus, $S$. intermedius, S. oralis, S. pneumoniae, S. mitis, S. sanguinis, S. gordonii, S. mutans, S. salivarius and S. bovis). Our results are in agreement with their conclusions. The three species of the 'anginosus' group clustered together. S. mitis, S. oralis and S. pneumoniae were highly related. S. sanguinis and S. gordonii clustered together. It is difficult to compare the other groupings because only a few species were included in the study of Teng et al. (2002). Hung et al. (2005) performed a phylogenetic analysis based on groEL gene sequence comparison of the 'mutans' group streptococci (S. sobrinus, S. downei, S. criceti, S. ratti and $S$. mutans). All species clustered together, but with a nonsignificant bootstrap value of $56 \%$, and two subclusters were noted. The first included the 'criceti' group and the second included S. mutans and S. ratti.

rpoB, sodA, groEL, gyrB and 16S rRNA gene-based phylogenetic trees inferred by using the neighbour-joining method were compared. The 'equinus' group was found in all the phylogenetic analyses. This cluster included four species, S. gallolyticus, S. infantarius, S. equinus and S. alactolyticus, and the bootstrap value found for the representative node was significant except in the phylogenies inferred from $16 \mathrm{~S}$ rRNA and gyrB sequence analysis. $S$. equinus clustered with $S$. infantarius with significant bootstrap values of $91-100 \%$. The 'salivarius' group and the 'hyovaginalis' group were always found (bootstrap values $99-100$ and $92-100 \%$, respectively). The 'criceti' group was always found with a significant bootstrap value (90-100\%) except in the 16S rRNA gene sequence analysis $(50 \%)$. The 'pyogenes' group was also found except in the phylogenetic analysis inferred from 16S rRNA gene sequence comparison. S. constellatus, S. intermedius and S. anginosus clustered together (bootstrap value 99-100 \%), generally in the 'mitis' cluster, except in the tree obtained by $16 \mathrm{~S}$ rRNA gene sequence comparison. The organization of the other representatives of the 'mitis' group was variable.

Recently, S. equinus and S. bovis were included as the same species, S. equinus (Schlegel et al., 2003). The two strains clustered together in all the phylogenetic analyses except for those from $g y r B$ gene sequence comparisons.

16S rRNA gene sequence similarities from type strains of different species ranged from $88.8 \%$ (S. macacae/S. acidominimus) to $99.7 \%$ ( $S$. thermophilus/S. salivarius), sodA sequence similarities ranged from $63.6 \%$ (S. massi- 


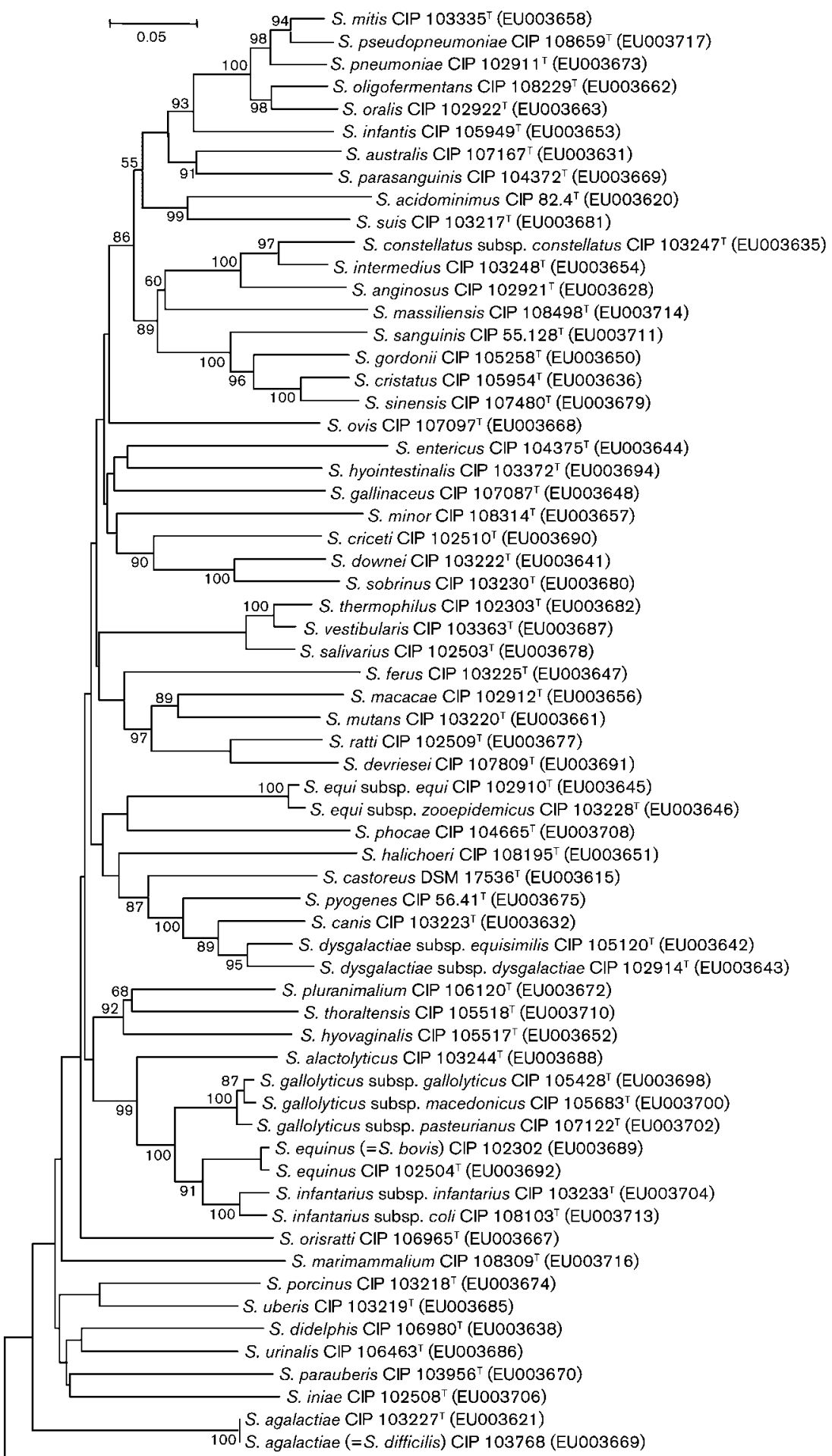

Fig. 1. Phylogenetic tree of members of the genus Streptococcus inferred from comparison of groEL gene sequences using the neighbour-joining method. Bar, 0.05 nucleotide changes per nucleotide position. The sequence of Gemella morbillorum CIP $81.10^{\top}$ was used as the outgroup. Bootstrap values $\geqslant 50$ are indicated.
liensis/S. dysgalactiae subsp. dysgalactiae) to $100 \%$ ( $S$. pneumoniae/S. pseudopneumoniae), rрoB sequence similarities ranged from $76.1 \%$ (S. sanguinis/S. marimammalium) to $97.6 \%$ ( $S$. thermophilus/S. vestibularis), gyrB sequence similarities ranged from $64.6 \%$ ( $S$. phocae/S. downei) to $97.5 \%$ ( $S$. constellatus/S. intermedius) and groEL sequence similarities ranged from $72.6 \%$ (S. sobrinus/S. agalactiae, S.
entericus/S. iniae) to $96.6 \%$ (S. thermophilus/S. vestibularis). Supplementary Fig. S3 shows the percentage similarity between each pair of strains for each gene; it shows that $r p o B$ is the least variable gene $(92.5 \%$ of the values are between 80 and $90 \%$ ) and $g y r B$ is the most variable $(82.1 \%$ of the values are between 70 and $80 \%$ and $15 \%$ are $<70 \%)$. 
16S rRNA gene sequence similarities from two subspecies ranged from $97.6 \%$ (S. gallolyticus subsp. gallolyticus/S. gallolyticus subsp. macedonicus) to $99.9 \%$ ( $S$. infantarius subsp. infantarius/S. infantarius subsp. coli), sodA sequence similarities ranged from $88.0 \%$ (S. dysgalactiae subsp. dysgalactiae/S. dysgalactiae subsp. equi) to $98.9 \%$ (S. equi subsp. equilS. equi subsp. zooepidemicus), rpoB sequence similarities ranged from $97.9 \%$ ( $S$. dysgalactiae subsp. dysgalactiae/S. dysgalactiae subsp. equi) to $99.6 \%$ ( $S$. gallolyticus subsp. gallolyticus/S. gallolyticus subsp. macedonicus), gyrB sequence similarities ranged from $93.7 \%$ ( $S$. infantarius subsp. infantarius/S. infantarius subsp. coli) to $98.5 \%$ (S. gallolyticus subsp. gallolyticus/S. gallolyticus subsp. macedonicus) and groEL sequence similarities ranged from $93.9 \%$ (S. dysgalactiae subsp. dysgalactiaelS. dysgalactiae subsp. equi) to $98.8 \%$ (S. gallolyticus subsp. gallolyticus/S. gallolyticus subsp. macedonicus). Based on subspecies type strain sequence comparisons, the similarity is lowest for sodA and gyrB. Moreover, in the phylogenies inferred from $\operatorname{sod} A$ and $\operatorname{gyr} B$ gene sequence comparisons, the two subspecies of $S$. infantarius did not cluster together. Also, in the phylogeny inferred from sodA gene sequence comparison, the two subspecies of $S$. dysgalactiae did not cluster together. In general, groEL is the best tool for differentiating subspecies: the percentage of similarity is discriminatory and clustering of subspecies is always obtained in the phylogenetic analysis.

Phylogenetic analysis and molecular identification based on only one gene is not recommended because of possible gene duplication, lateral gene transfer or gene loss, which can distort the results. Partial sequencing of the $16 \mathrm{~S}$ rRNA gene is the first step in bacterial strain identification. It allows identification at the genus level and the recognition of novel species if the similarity between the new isolate and recognized species is $\leqslant 97 \%$ (Stackebrandt et al., 2002). Housekeeping genes are also suitable for identification at the species or subspecies level, as demonstrated for several bacterial phyla (Holmes et al., 2004; Martens et al., 2008; Naser et al., 2007). In this study, the use of $\operatorname{sodA}$, $r p o B, g y r B$ and groEL gene sequence comparisons allowed us to improve our knowledge of the taxonomy and phylogenetic relationships of the genus Streptococcus. Presently, sodA and $r p o B$ gene sequence comparisons are the tools used to identify strains at the species level in the genus Streptococcus. gyrB has been shown to be a good tool for identifying some members of the genera Aeromonas (Küpfer et al., 2006), Salmonella, Shigella, Escherichia (Fukushima et al., 2002) and Pseudomonas (Radice et al., 2006). At present, the phylogeny obtained from gyrB gene sequence comparison presents some inconsistencies. groEL has also been described as a good tool for identification in the genera Rickettsia (Lee et al., 2003), Campylobacter (Kärenlampi et al., 2004), Lactobacillus (Blaiotta et al., 2008) and Bacillus (Chang et al., 2003). In our study, which included representatives of most species of the genus Streptococcus for the first time, groEL gene sequence comparison was a powerful tool for establishing phylogeny and differentiating Streptococcus species. However, for each species, sequences will have to be obtained from several isolates in order to confirm the results established by this study of type strains.

\section{References}

Bentley, R. W., Leigh, J. A. \& Collins, M. D. (1991). Intrageneric structure of Streptococcus based on comparative analysis of smallsubunit rRNA sequences. Int J Syst Bacteriol 41, 487-494.

Blaiotta, G., Fusco, V., Ercolini, D., Aponte, M., Pepe, O. \& Villani, F. (2008). Lactobacillus strain diversity based on partial hsp60 gene sequences and design of PCR-restriction fragment length polymorphism assays for species identification and differentiation. Appl Environ Microbiol 74, 208-215.

Chang, Y. H., Shangkuan, Y. H., Lin, H. C. \& Liu, H. W. (2003). PCR assay of the groEL gene for detection and differentiation of Bacillus cereus group cells. Appl Environ Microbiol 69, 4502-4510.

Drancourt, M., Roux, V., Fournier, P. E. \& Raoult, D. (2004). rpoB gene sequence-based identification of aerobic Gram-positive cocci of the genera Streptococcus, Enterococcus, Gemella, Abiotrophia and Granulicatella. J Clin Microbiol 42, 497-504.

Facklam, R. (2002). What happened to the streptococci: overview of taxonomic and nomenclature changes. Clin Microbiol Rev 15, 613630.

Farris, J. S. (1970). Methods for computing Wagner trees. Syst Zool 19, 83-92.

Felsenstein, J. (1989). PHYLIP - phylogeny inference package (version 3.2). Cladistics 5, 164-166.

Fukushima, M., Kakinuma, K. \& Kawaguchi, R. (2002). Phylogenetic analysis of Salmonella, Shigella, and Escherichia coli strains on the basis of the gyrB gene sequence. J Clin Microbiol 40, 2779-2785.

Garnier, F., Gerbaud, G., Courvalin, P. \& Galimand, M. (1997). Identification of clinically relevant viridans group streptococci to the species level by PCR. J Clin Microbiol 35, 2337-2341.

Gil, R., Silva, F. J., Pereto, J. \& Moya, A. (2004). Determination of the core of a minimal bacterial gene set. Microbiol Mol Biol Rev 68, 518537.

Holmes, D. E., Nevin, K. P. \& Lovley, D. R. (2004). Comparison of $16 \mathrm{~S}$ rRNA, nifD, recA, gyrB, rpoB and fusA genes within the family Geobacteraceae fam. nov. Int J Syst Evol Microbiol 54, 15911599.

Hoshino, T., Fujiwara, T. \& Kilian, M. (2005). Use of phylogenetic and phenotypic analyses to identify nonhemolytic streptococci isolated from bacteremic patients. J Clin Microbiol 43, 6073-6085.

Hung, W. C., Tsai, J. C., Hsueh, P. R., Chia, J. S. \& Teng, L. J. (2005). Species identification of mutans streptococci by groESL gene sequence. J Med Microbiol 54, 857-862.

Igarashi, T., Ichikawa, K., Yamamoto, A. \& Goto, N. (2001). Identification of mutans streptococcal species by the PCR products of the dex genes. J Microbiol Methods 46, 99-105.

Itoh, Y., Kawamura, Y., Kasai, H., Shah, M. M., Nhung, P. H., Yamada, M., Sun, X., Koyana, T., Hayashi, M. \& other authors (2006). dnaJ and gyrB gene sequence relationship among species and strains of genus Streptococcus. Syst Appl Microbiol 29, 368-374.

Kärenlampi, R. I., Tolvanen, T. P. \& Hänninen, M. L. (2004). Phylogenetic analysis and PCR-restriction fragment length polymorphism identification of Campylobacter species based on partial groEL gene sequences. J Clin Microbiol 42, 5731-5738. 
Kawamura, Y., Whiley, R. A., Shu, S. E., Ezaki, T. \& Hardie, J. M. (1999). Genetic approaches to the identification of the mitis group within the genus Streptococcus. Microbiology 145, 2605-2613.

Kawamura, Y., Itoh, Y., Mishima, N., Ohkusu, K., Kasai, H. \& Ezaki, T. (2005). High genetic similarity of Streptococcus agalactiae and Streptococcus difficilis: S. difficilis Eldar et al. 1995 is a later synonym of S. agalactiae Lehmann and Neumann 1896 (Approved Lists 1980). Int J Syst Evol Microbiol 55, 961-965.

Kimura, M. (1980). A simple method for estimating evolutionary rates of base substitutions through comparative studies of nucleotide sequences. J Mol Evol 16, 111-120.

Küpfer, M., Kuhnert, P., Korczak, B. M., Peduzzi, R. \& Demarta, A. (2006). Genetic relationships of Aeromonas strains inferred from $16 \mathrm{~S}$ rRNA, gyrB and $r p o B$ gene sequences. Int J Syst Evol Microbiol 56, 2743-2751.

Lee, J. H., Park, H. S., Jang, W. J., Koh, S. E., Kim, J. M., Shim, S. K., Park, M. Y., Kim, Y. W., Kim, B. J. \& other authors (2003). Differentiation of rickettsiae by groEL gene analysis. J Clin Microbiol 41, 2952-2960.

Martens, M., Dawyndt, P., Coopman, R., Gillis, M., De Vos, P. \& Willems, A. (2008). Advantages of multilocus sequence analysis for taxonomic studies: a case study using 10 housekeeping genes in the genus Ensifer (including former Sinorhizobium). Int J Syst Evol Microbiol 58, 200-214.

Naser, S. M., Dawyndt, P., Hoste, B., Gevers, D., Vandemeulebroecke, K., Cleenwerck, L., Vancanneyt, M. \& Swings, J. (2007). Identification of lactobacilli by pheS and rpoA gene sequence analyses. Int J Syst Evol Microbiol 57, 2777-2789.

Picard, F. J., Ke, D., Boudreau, D. K., Boissinot, M., Huletsky, A., Richard, D., Ouellette, M., Roy, P. H. \& Bergeron, M. G. (2004). Use of tuf sequences for genus-specific PCR detection and phylogenetic analysis of 28 streptococcal species. J Clin Microbiol 42, 3686-3695.

Poyart, C., Quesne, G., Coulon, S., Berche, P. \& Trieu-Cuot, P. (1998). Identification of streptococci to species level by sequencing the gene encoding the manganese-dependent superoxide dismutase. $J$ Clin Microbiol 36, 41-47.

Radice, F., Orlandi, V., Massa, V., Cavalca, L., Demarta, A., Wood, T. K. \& Barbieri, P. (2006). Genotypic characterization and phylogenetic relations of Pseudomonas sp. (formerly P. stutzeri) OX1. Curr Microbiol 52, 395-399.

Saitou, N. \& Nei, M. (1987). The neighbor-joining method: a new method for reconstructing phylogenetic trees. Mol Biol Evol 4, 406425.

Schlegel, L., Grimont, F., Ageron, E., Grimont, P. A. D. \& Bouvet, A. (2003). Reappraisal of the taxonomy of the Streptococcus bovis/ Streptococcus equinus complex and related species: description of Streptococcus gallolyticus subsp. gallolyticus subsp. nov., S. gallolyticus subsp. macedonicus subsp. nov. and S. gallolyticus subsp. pasteurianus subsp. nov. Int J Syst Evol Microbiol 53, 631-645.

Stackebrandt, E., Frederiksen, W., Garrity, G. M., Grimont, P. A. D., Kämpfer, P., Maiden, M. C. J., Nesme, X., Rosselló-Mora, R., Swings, J. \& other authors (2002). Report of the ad hoc committee for the re-evaluation of the species definition in bacteriology. Int $J$ Syst Evol Microbiol 52, 1043-1047.

Tamura, K., Dudley, J., Nei, M. \& Kumar, S. (2007). MEGA4: molecular evolutionary genetics analysis (MEGA) software version 4.0. Mol Biol Evol 24, 1596-1599.

Tapp, J., Thollesson, M. \& Herrmann, B. (2003). Phylogenetic relationships and genotyping of the genus Streptococcus by sequence determination of the RNase P RNA gene, rnpB. Int J Syst Evol Microbiol 53, 1861-1871.

Teng, L. J., Hsueh, P. R., Tsai, J. C., Chen, P. W., Hsu, J. C., Lai, H. C., Lee, C. N. \& Ho, S. W. (2002). groESL sequence determination, phylogenetic analysis, and species differentiation for viridans group streptococci. J Clin Microbiol 40, 3172-3178.

Thompson, J. D., Gibson, T. J., Plewniak, F., Jeanmougin, F. \& Higgins, D. G. (1997). The CLUSTAL_X windows interface: flexible strategies for multiple sequence alignment aided by quality analysis tools. Nucleic Acids Res 25, 4876-4882.

Weisburg, W. G., Barns, S. M., Pelletier, D. A. \& Lane, D. J. (1991). $16 \mathrm{~S}$ ribosomal DNA amplification for phylogenetic study. J Bacteriol 173, 697-703.

Zeigler, D. R. (2003). Gene sequences useful for predicting relatedness of whole genomes in bacteria. Int J Syst Evol Microbiol 53, 1893-1900. 